Project 09-774

\title{
Quantifying Water Extraction by TBP/Dodecane Via Molecular Dynamics Simulations
}

\section{Integrated University Programs}

Dr. Bamin Khomami

University of Tennessee, Knoxville

In collaboration with:

Oak Ridge National Laboratory

Terry Todd, Technical POC

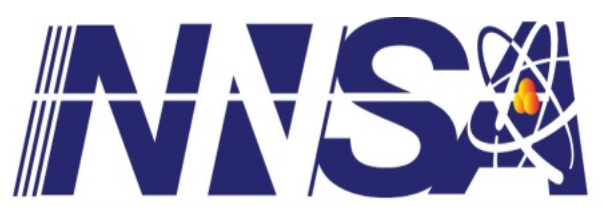




\title{
Final Project Report
}

Project Title: Quantifying Water Extraction by TBP/Dodecane via Molecular Dynamics Simulations

Recipient: University of Tennessee at Knoxville

Award Number: DE-AC07-051D14517

Project Number: 09-774

Principal Investigators:

Bamin Khomami; Phone: 865974 2035; e-mail: bkhomami@utk.edu.

Shengting Cui; Phone: 865-974-4820; e-mail: scui@utk.edu

Collaborators at the Oak Ridge National Laboratory:

Valmor F. de Almeida, dealmeidav@ornl.gov.

Benjamin P. Hay, haybp@ornl.gov.

Kevin Felker, felkerlk@ornl.gov

\section{(a) Project Objective and Results}

\begin{abstract}
Objective: The purpose of this project is to quantify the interfacial transport of water into the most prevalent nuclear reprocessing solvent extractant mixture, namely tri-butylphosphate (TBP) and dodecane, via massively parallel molecular dynamics simulations on the most powerful machines available for open research. Specifically, we will accomplish this objective by evolving the water/TBP/dodecane system up to 1 ms elapsed time, and validate the simulation results by direct comparison with experimentally measured water solubility in the organic phase. The significance of this effort is to demonstrate for the first time that the combination of emerging simulation tools and state-of-the-art supercomputers can provide quantitative information on par to experimental measurements for solvent extraction systems of relevance to the nuclear fuel cycle.
\end{abstract}

Efforts and Results: To achieve the object described above, initially, the single component, single phase systems were studied to verify existing and develop new molecular models, followed by a single phase mixture, and then finally the complete twophase water extraction system. Specifically, the systems we studied are: (1) pure TB; (2) n-doedecane; (3) TBP/n-dodecane mixture; and (4) the complete extraction system: water-TBP/n-dodecane two phase system to gain deep insight into the water extraction process. We have completely achieved our goal of extracting water molecules into the TBP/n-dodecane mixture to saturation and obtained favorable comparison with experiment. Many insights into fundamental molecular level processes and physics were obtained from the process. Most importantly, we found that the dipole moment of the 
extracting agent is crucially important in affecting the interface roughness and the extraction rate of water molecules into the organic phase. In addition, we have identified shortcomings in the existing OPLS-AA force field potential for long-chain alkanes. The significance of this force field is that it is supposed to be optimized for molecular liquid simulations. We found that it failed for n-dodecane and/or longer chains to correctly predict the liquid state and thus not usable for our water extraction simulation. We have proposed a simple modification to circumvent the artificial crystallization of the long alkane chains applicable at ambient condition.

\section{(b) Effort performed and the accomplishments achieved}

\section{TBP Force Field Model Development}

We conducted force field survey on 4 sets of force field parameters for TBP, a molecule composed of a phosphate head group and three butyl tails as shown in Figure 1 . The 4 sets of parameters are based on adoption of Van der Waals parameters for the phosphoryl head group and the butyl tail group of the TBP molecules, from separate force field models already in use. In particular, we used the Amber parameters for the Van der Waals interaction of the phosphoryl group, combined with either the Amber or the OPLSAA (Optimized Potential for Liiquid Simulation-All Atoms) force field for the butyl tail groups. For the intramolecular force parameters, we used the parameter sets from the Amber force field. Two sets of atomic partial charges have been examined. One was that has been previously used by other researchers which was derived from a semi-empirical quantum chemistry calculation-MNDO (Modified Neglect of Differential Overlap), and the other derived from our own quantum chemistry calculation using the density functional theory (DFT). The four set of force field parameters are thus designated as OPLS-MNDO, OPLS-DFT, Amber-MNDO, Amber-DFT to reflect the combination of the Van der Waals and charge parameters used. Using the original atomic partial charges, the MNDO charge model predicts a TBP electric dipole moment about 4.9 D, whereas our DFT charge model predicts a TBP dipole moment of about $3.8 \mathrm{D}$, which is much closer to the experimental values of 3.07-3,32 D [1,2]. Thus, we have attempted to rescale the atomic partial charges to reproduce the experimentally measured electric dipole moment of the TBP molecules in liquid state. Using a scale factor 0.7 , the DFT charge set predicts a TBP dipole moment $3.0 \mathrm{D}$, while a scale factor 0.6 has to be used to reproduce a value comparable to experiment, $3.2 \mathrm{D}$. All the force fields give reasonable mass density prediction with deviation within 3\% from experiment [3]. The better performing parameter sets, OPLS-MNDO predicts the mass density to within $0.5 \%$, and the OPLS-DFT predicts the mass density to within $1 \%$. The details are described in a paper published in the Journal of Physical Chemistry 116, 305-313 (2012).
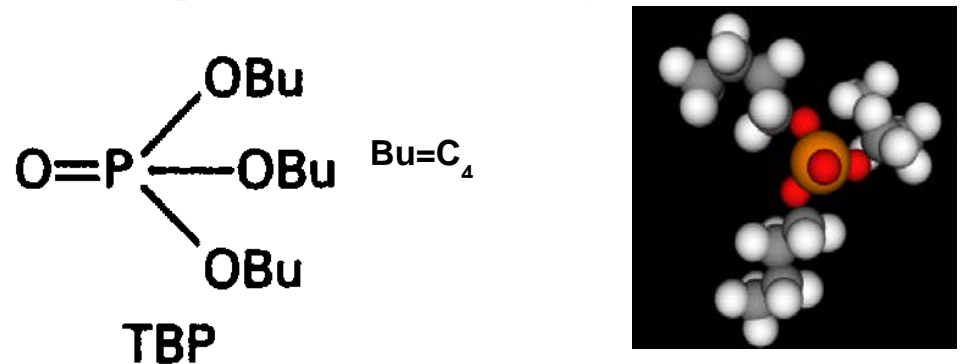

Figure 1 Schematic of TBP molecule. 


\section{N-doecane Force Field Development}

Although the force field parameters currently in use in molecular modeling were developed through $a b$ initio electronic structure calculations and carefully calibrated with experimental data for prototypical molecular entities, less well-founded choices have been made for some of the non-bonded intramolecular interactions, such as the 1-4 intramolecular Van der Waals and Coulombic interactions. In the OPLS-AA force field model, a scaling factor of $1 / 2$ was recommended [4]. When applied to n-dodecane, we discovered that the standard OPLS-AA with the standrd scaling factor leads to a quasicrystalline phase at ambient conditions where a disordered liquid state is expected (Figure 2). We have traced the physical origin of this tendency for crystallization to be too stiff a chain backbone of the n-dodecane for which the scaling factor is a significant contributor through the intramolecular interaction of the 1-4 atom pair along the chain.

We conducted extensive calculations of the n-alkanes in the range of carbon number from C10 to C16, to investigate the chain length variation of such effect. While it appears that in OPLS-AA the standard scaling factor of $1 / 2$ works correctly for carbon number less or equal to 10 , for carbon number equal or greater than 12, the effect becomes progressively worse. Beyond carbon number 12, as the chain length increases, the scaling factor has to be decreased for each carbon number increase to keep the n-alkanes in the liquid state, expected based on experimental data. For n-hexadecane, the scaling factor has to be reduced to 0 to be consistent with experiment. Too ascertain that our results are not an artifact of improper equilibration or system size effect, we have conducted the simulations for long time evolutions and with varying initial configurations. We have also carried out simulations with atom number almost an order of magnitude larger which all gave consistent results. Interestingly, during the process of our manuscript publication, a research group in Austria independently published a paper confirming our results.

We found that all the thermophysical properties, including: the heat of vaporization, selfdiffusion coefficient, melting point, and gauche/trans ratio are all systematically improved with the decrease of the SF. The mass density is essentially invariant below SF $=0.4$ and in close agreement with experiment. For SF $=0$, the lowest SF possible, the calculated heat of vaporization is $63.47 \pm 0.59 \mathrm{~kJ} / \mathrm{mol}$ compared to the experimental value of $61.287 \mathrm{~kJ} / \mathrm{mol}$. The calculated melting point is $261 \pm 1 \mathrm{~K}$ compared to $263.356 \mathrm{~K}$ in experiment. The calculated self-diffusion coefficient is $(5.747 \pm 0.156) \times 10^{-6} \mathrm{~cm}^{2} / \mathrm{s}$, somewhat lower than the experimental value of $8.71 \times 10^{-6} \mathrm{~cm}^{2} / \mathrm{s}$. These predictions are comparable to the best prediction in literature and for diffusion coefficient, is slightly better than existing models. Note that this is a huge improvement over the original OPLSAA model with the standard SF which predicts a self-diffusion coefficient of zero due to the solidification. The results were published in the Journal of Molecular Modeling 19, 1251 (2012). 


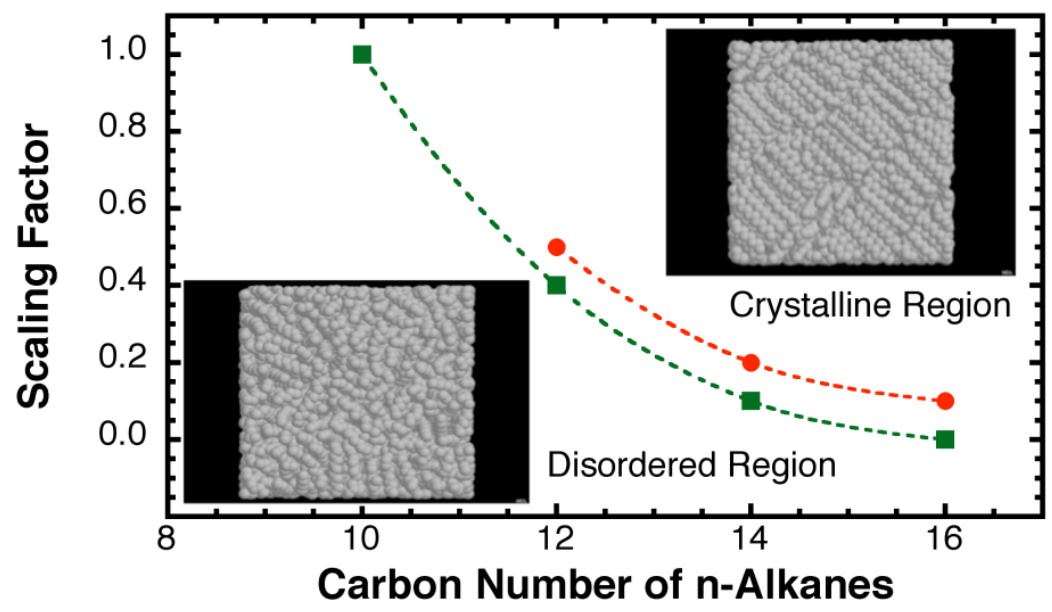

Figure 2 The phase boundary for $n$-alkanes as determined by the scaling factor.

\section{TBP/n-Dodecane Mixture}

We have carried out extensive calculations on the mixture of n-dodecane and TBP, using the modified OPLS model for n-dodecane (with the 1-4 scaling factor equal to 0.3) and the two best performing force fields for TBP: OPLS-MNDO and OPLS-DFT, using the standard Lorentz-Berthelot mixing rules for different atomic species. We have used the rescaled atomic charges (scaling factor 0.7 for DFT charges and 0.6 for MNDO charges) so that they correctly reproduce the experimentally measured electric dipole moment of TBP. Our work on pure TBP has found that the rescaled charge models perform better than the original charges in predicting the thermophysical properties of TBP.

\section{(1) Mass Density and Excess Volume of Mixing}

The calculated mass density of the mixture is in excellent agreement with experiment, to within about $1 \%$ for the OPLS-DFT model, and $0.75 \%$ for the OPLS-MNDO model in the entire mole fraction range $0.0-1.0$. The excess volume of mixing is positive in the entire range of mole fraction, also in agreement with experiment. The experimentally measured excess volume peaks around mole fraction 0.5, while the calculated excess volume peaks between TBP mole fraction 0.3 to 0.5 due to statistical uncertainty, in reasonable agreement with experiment considering the smallness of the excess volume of mixing $\sim 0.9 \mathrm{~cm}^{3} / \mathrm{mol}$, which is less than $0.4 \%$ of the molar volume of either n-dodecane or TBP. We attribute this positive volume of mixing to the disparity between the molecular shape of the n-dodecane (line shape) and TBP (approximately globular). When two molecular species with large shape disparity mix together, relatively large vacancies are created so the mixing volume increases.

\section{(2) Variation of Dipole Moment of TBP with Concentration}

The calculated dipole moment of TBP in the mixture slightly increases with the TBP concentration, from 2.84 to 2.99 D, or about $5.3 \%$ for the OPLS-DFT model; from 3.10 to $3.23 \mathrm{D}$, or about $4.2 \%$ for the OPLS-MNDO model in the mole fraction range 0 to 1 . This is comparable to experimental observation, where a similar increase was found 
$[1,2,5]$. In dilute solution of alkane solvent, the measured TBP dipole moment falls in the range between 2.6-2.92 [5], while in pure TBP, it ranges between 3.07 to $3.32 \mathrm{D}$ [1,2].

\section{(3) Dimerization of TBP in Solution}

In the literature, there has been the suggestion for a possible self-association of TBP in the form of dimers in solution. This has been supported with a variety of experiments, such as, distribution coefficient experiment, infrared spectroscopy, nuclear magnetic resonance, calorimetry, and vapor pressure osmometry [5,6]. We have calculated a variety of spatial pair correlation functions between atoms of two TBP molecules in the solution. Through analyzing the spatial pair correlation functions between P-P, P-O2, and $\mathrm{O} 2-\mathrm{O} 2$ of the phosphoryl head group, we find that TBP molecules indeed have a most probable arrangement of two $\mathrm{P}=\mathrm{O} 2$ dipoles forming a anti-parallel configuration, with the two pairs of $\mathrm{P}$ and $\mathrm{O} 2$ atoms constituting a parallelogram. Our calculated peak positions of the P-P, P-O2, and O2-O2 suggest a P-P distance of about $0.61 \mathrm{~nm}$, and an O2-P-O2 angle about $74^{\circ}$. The acute O2-P-O2 angle is consistent with an anti-parallel arrangement of the two $\mathrm{P}=\mathrm{O} 2$ bonds that favor the electrostatic attraction between the two electric dipoles as dictated by the fundamental laws of physics.

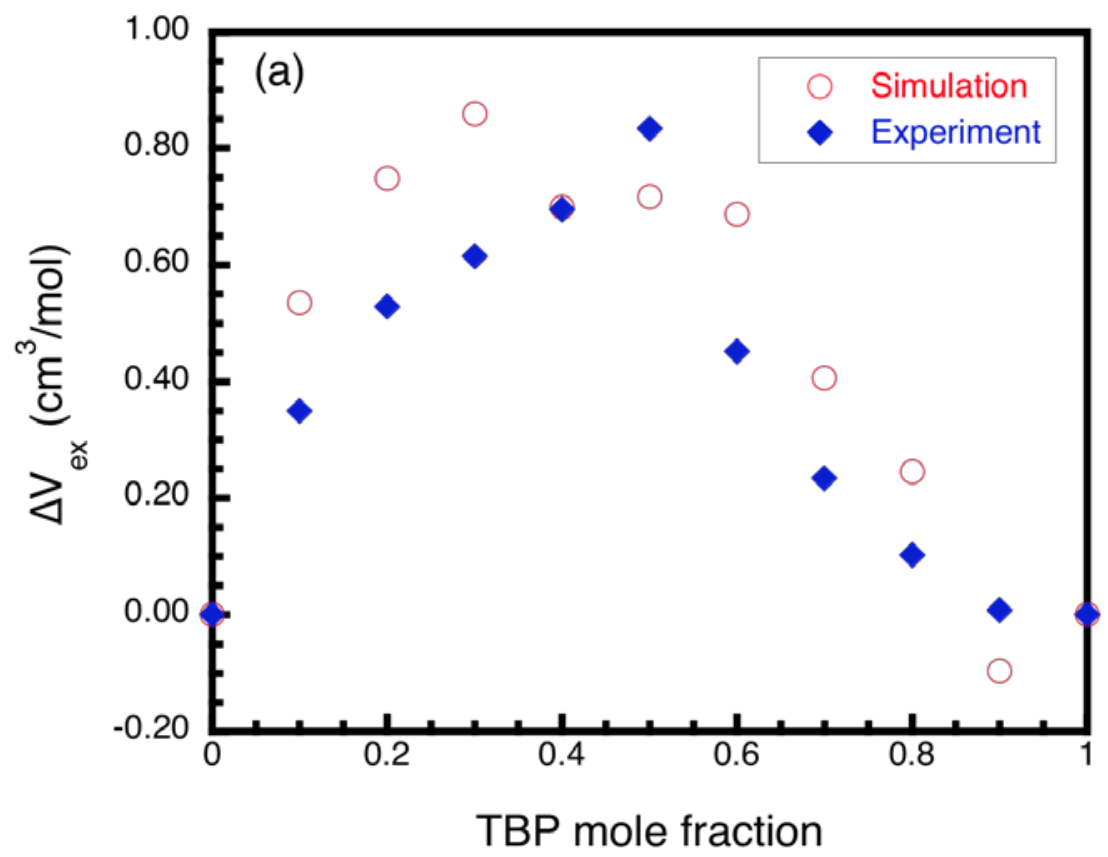

Figure 3 Excess volume of mixing for the TBP/n-dodecane mixture as a function of the mole fraction for the DFT charge model. Open circles are for simulation, and the solid diamonds are from experiment.

\section{Water Extraction}

We have mostly used a rescaled charge of 0.9 times of the original DFT-based atomic charge, to perform the water extraction which gives a very close prediction of the solubility of water into the mixture of TBP and dodecane when compared to experimental results. This charge model also predicts a dipole moment for the TBP molecules, $3.54 \mathrm{D}$, 
which is close to the experimental value of 3.07-3.32 D. Thus this is a physically based model for predicting water extraction from the aqueous phase to the organic phase.

In the water extraction simulations, the following systems have been studied.

(1) System size: 89,000 atoms, the original DFT-based charge: We have evolved the system for $140 \mathrm{~ns}$, at which point we have observed an extended plateau of $10.8 \mathrm{~g} / \mathrm{L}$ spanning $70 \mathrm{~ns}$ for water solubility in the organic phase, compared to experimental value of $5.8 \mathrm{~g} / \mathrm{L}$ (see Figure $1 \mathrm{~b}$ in the attached manuscript). We have thus concluded that the original DFT charge for TBP molecules over predicts the water solubility in the organic phase. No further calculation was performed for this system.

(2) System size: 89,000 atoms, rescaled DFT charge by a factor 0.9: We evolved this system up to about 140 ns. The water concentration in the organic phase reached saturation at about 70 ns (see Figure 2 in the attached manuscript). Thus, there is an extended period of time where water concentration in the organic phase fluctuate around the average value, $4.5 \mathrm{~g} / \mathrm{L}$. Such extended plateau suggests the system has reach equilibrium, and the solubility obtained is reasonably close to the experimental value of $5.8 \mathrm{~g} / \mathrm{L}$.

(3) System size: 89,000 atoms, rescaled DFT charge by a factor 0.8: For this calculation, we performed the MD simulation up to about $85 \mathrm{~ns}$, the water concentration in the organic phase reached a plateau of $\sim 0.5 \mathrm{~g} / \mathrm{L}$, at the simulation time of $35 \mathrm{~ns}$, which is too low (see Figure 2 in the attached manuscript). This indicates that the water solubility is sensitively dependent on the atomic charge (or equivalently, the dipole moment) of the TBP molecules.

In summary, the aforementioned large scale simulations clearly suggest that the amphiphilicity of the extraction agent plays a crucial in the extraction rate.

(4) System size: 360,000 atoms, rescaled DFT charge by a factor 0.9:This simulation started from a previous calculation where an original MNDO-based model was used which over predicted the saturated water solubility in the organic phase, giving a solubility $\sim 12 \mathrm{~g} / \mathrm{L}$ compared to about $5.8 \mathrm{~g} / \mathrm{L}$ in experiment. The system was then evolved using the DFTbased model with corresponding atomic charges rescaled by a factor of 0.9 for about 52 ns. It was found that the extracted water solubility in the organic phase decreased from $\sim 12 \mathrm{~g} / \mathrm{L}$ to $\sim 8.0 \mathrm{~g} / \mathrm{L}$. We observed a short transient plateau from 41 ns to $52 \mathrm{~ns}$, further decrease was observed from 52 ns to 65 ns. Comparing this calculation with that described in (2), we expect the water solubility in the organic phase to vary between 4.7 $\mathrm{g} / \mathrm{L}$ and $8.0 \mathrm{~g} / \mathrm{L}$. The experimentally observed solubility falls in this range which suggests the current model employed could realistically predict the water extraction through liquid-liquid interface and the equilibrium solubility in the organic phase. 

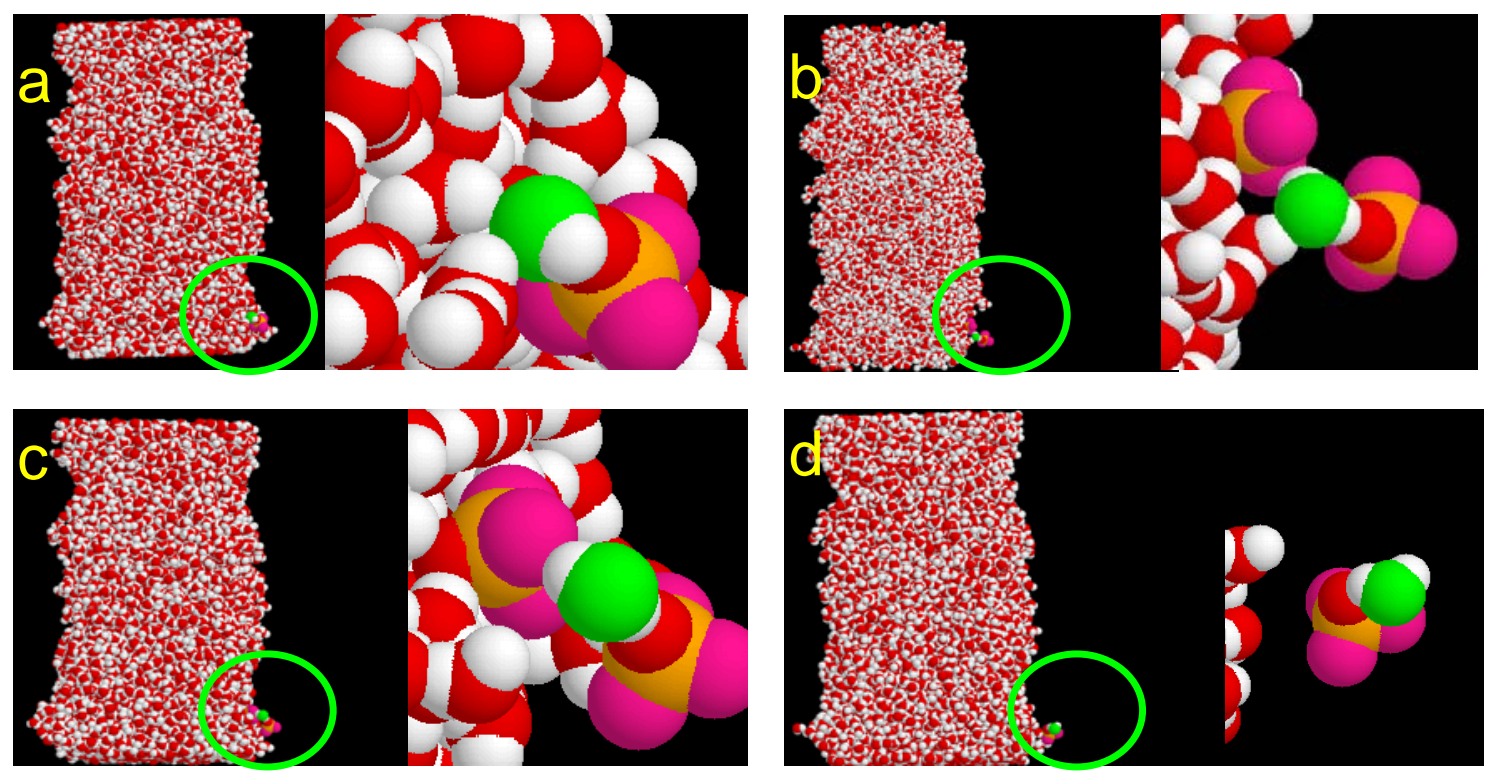

Figure 4 Water extracted as a single water molecule by one TBP molecule with the assistance of another TBP molecule. Snapshots (a) to (d) are for $80.030 \mathrm{~ns}$ (a), $80.040 \mathrm{~ns}$ (b), $85.045 \mathrm{~ns}$ (c), and 80.050ns (d), respectively. The oxygen of the water being monitored is in green, and the hydrogen of the monitored water is in white. Otherwise, the oxygen of water is in red, and the hydrogen of water is in white. For TBP, only the TBP head group is shown, $\mathrm{O} 2$ is in red, $\mathrm{P}$ is in yellow, and OS is in pink. In all snapshots, n-dodecane is not shown.

The molecular simulations performed in this project in this project has provided a wealth of general guidelines as well as molecular level insight into the extraction process. Specifically, it has been clearly demonstrated tha the TBP electric dipole moment has a significant effect on the predicted water solubility. By scaling down the original DFT-based atomic partial charges of the TBP molecular model by 0.9 , water solubility close to that measured in experiments was satisfactorily predicted.

We have also demonstrated that a higher TBP dipole moment increases the roughness of the water/organic interface as a result of reduced interfacial tension (or equivalently, the decreased free energy required for interface area expansion). Because of a rougher interface, more water molecules are exposed to the organic phase, and located at the edges or tips of the aqueous-organic interface with fewer hydrogen bonds connected to the aqueous phase. This creates a favorable environment for water hydrogen bond breaking with the bulk water and subsequent transfer to the organic phase.

Overall, the simulations clearly indicate that in the water extraction process, the critical step is the hydrogen bond breaking of interfacial water molecules. In this step, TBP plays a critical role in a) forming hydrogen bond with hydrogen atoms of interfacial water, b) preventing water molecules' dangling bonds to reconnect with bulk water, and c) providing a driving force for finally breaking the weakly bonded 
single hydrogen bond of water or water clusters. (Figure 4 shows one example of a single water molecule being extracted into the organic phase.) Thus, the interface coverage by TBP molecules is crucial both in increasing the interfacial roughness and providing the driving force for extracting water into the organic phase. It is therefore important to understand the interfacial transport properties of extracting agents, in addition to their chemical binding properties, when considering practical applications.

Water molecules are extracted as single water molecules or as various sized water clusters with TBP attached to them. The majority of extracted water forms water clusters in the bulk organic phase, however the majority of these clusters are smaller than 5 water molecules when the extraction process reaches saturation (see Figure 9 of the attached manuscript).

\section{References}

1. Estok, G.; Wendlandt, W. W. J. Am. Chem. Soc. 1955, 77, 4767.

2. Petkovic, D. M.; Kezele, B. A.; Rajic, D. R.; J. Phys. Chem. 1973, 77, 922.

3. Tian, Q.; Liu, H. J. Chem. Eng. Data 2007, 52, 892.

4. Thomas LL, Christakis TJ, and Jorgensen WL (2006) J. Phys. Chem. B 110, 2119821204.

5. Schultz, W. W.; Navratil, J. S.; Eds.; The Science and technology of tributyl phosphate, Vol. 1, CRC Press, Boca Raton, FL, 1984.

6. Motokawa, R.; Suzuki, S.; Ogawa, H.; Mark R. Antonio, M. R.; Yaita, T. J. Phys. Chem. B 2012, 116, 1319.

\section{(c) List of any publication or information release made of material developed or maintained through the performance of the subcontract}

\section{Conference Presentations:}

2012 AIChE Annual Meeting: Pittsburgh, PA: Xianggui Ye, Shengting Cui, Bamin Khomami, Valmor F de Almeida, 'Molecular Dynamics Simulation of Interfacial Water Extraction by TBP/n-Dodecane.”

2011 AIChE Annual Meeting: Minneapolis, MN: Xianggui Ye, Shengting Cui, Bamin Khomami, Valmor F de Almeida, "Atomistic Simulation of Water Extraction by TBP/nDodecane Solution.”

2010 AIChE Annual Meeting: Salt Lake City, UT: Xianggui Ye, Shengting Cui, and Bamin Khomami, Valmor de Almeida, and Benjamin Hay, "Uranyl Nitrate Complex Migration into TBP/Dodecane Organic Solution: A Molecular Dynamics Study.” 


\section{Journal Publications:}

1. Xianggui Ye, Shengting Cui, Valmor F. de Almeida, Benjamin P. Hay, and Bamin Khomami, "Uranyl nitrate complex extraction into TBP/dodecane organic solutions: a molecular dynamics study," Phys. Chem. Chem. Phys. 2010, 12, 15406-15409.

2. S. T. Cui, V. F. de Almeida, B. P. Hay, X. Ye., and B. Khomami, "Molecular Dynamics Simulation of Tri-n-butyl-Phosphate Liquid: A Force Field Comparative Study,” J. Phys. Chem. B, 2012, 116, 305-313.

3. Xianggui Ye, Shengting Cui, Valmor F. de Almeida, and Bamin Khomami, "Effect of Varying the 1-4 Intramolecular Scaling Factor in Atomistic Simulations of Long-Chain N-alkanes with the OPLS-AA Model,” J. Molecular Modeling, , 2012.

4. Xianggui Ye, Shengting Cui, Valmor F. de Almeida, and Bamin Khomami, "Molecular Simulation of Water Extraction into a Tri-n-Butyl-Phosphate/n-Dodecane Solution," J. Phys. Chem. B, Submitted (2013).

4. Manuscript ready for submission: Shengting Cui, Valmor F. de Almeida, and Bamin Khomami, "Molecular Dynamics Simulations of Tri-n-butyl-Phosphate/n-Dodecane Mixture: Thermophysical Properties and Molecular Structure,” J. Phys. Chem. B, to be submitted, October (2013).

\section{(d) Other Relevant Information}

The project supported one undergraduate student, Brian Smith, for one semester during his senior year. He has since graduated and entered University of Michigan Graduate School.

Another person this project trained is a postdoctoral researcher, Xianggui Ye. He has now gained a lot of expertise in the liquid-liquid separation of nuclear materials. 


\title{
Molecular Simulation of Water Extraction into a Tri-n-Butyl-Phosphate/n- Dodecane Solution
}

\author{
Xianggui Ye ${ }^{1}$, Shengting Cui ${ }^{1, \dagger}$, Valmor F. de Almeida ${ }^{2}$, and Bamin Khomami ${ }^{1, \dagger}$ \\ ${ }^{1}$ Materials Research and Innovative Laboratory (MRAIL), Department of Chemical \\ and Biomolecular Engineering, University of Tennessee, Knoxville, TN 37996 \\ ${ }^{2}$ Oak Ridge National Laboratory, Oak Ridge, TN 37831-6181, USA
}

\begin{abstract}
Molecular dynamics simulations were performed to investigate water extraction into a solution of $30 \mathrm{vol} \%$ tri- $n$-butyl-phosphate (TBP) in $n$-dodecane. Our computational results indicate that the TBP electric dipole moment has a significant effect on the predicted water solubility. A larger TBP dipole moment decreases the aqueous-organic interfacial tension, leading to increased roughness of the aqueous-organic interface. Interfacial roughness disrupts the interfacial water hydrogen bonding structure, resulting in a presence of dangling water molecules at the interface. The increased interfacial roughness enhances the probability of water molecules breaking away from the aqueous phase and migrating into the organic bulk phase. By varying the atomic partial charges of the TBP molecules to reproduce a dipole moment close to experimentally measured value, we were able to predict water solubility in close agreement with experimental measurements. In addition, our simulation results reveal the detailed molecular mechanism of water extraction process, and the various structural forms of water molecules both at the interface and in the bulk organic phase.
\end{abstract}

Keywords: Water extraction; Molecular dynamic simulation; Tri- $n$-Butyl-Phosphate; interfacial roughness, dipole moment.

\footnotetext{
† Corresponding authors: scui@utk.edu, dealmeidav@ornl.gov, and bkhomami@utk.edu
} 


\section{Introduction}

Solvent extraction is a commonly used process in hydrometallurgy and industrial nuclear fuel reprocessing. Metal cations, acid, and water are typically co-extracted from the aqueous phase into the organic phase, which is a mixture of an extracting agent, such as tri- $n$-butyl phosphate (TBP) (Schematic 1), and a diluent, such as $n$ dodecane. Among the different actinide separation methods, solvent extraction has several advantages at industrial scale, including the ability for continuous operation, high throughput and solvent recycling., ${ }^{1,2}$ Recently, an increased interest in recycling of used nuclear fuel has prompted us to carry out an investigation of uranyl extraction by molecular dynamics simulation which elucidated molecular level mechanisms for this extraction process. ${ }^{3-5}$ Specifically, we have found that the uranyl complexes are formed at the interface between the aqueous phase and the organic phase and the electrically neutral species are preferentially extracted into the organic phase. Our results further suggest that the experimentally found $\mathrm{UO}_{2}\left(\mathrm{NO}_{3}\right)_{2}$ (TBP $)_{2}$ complex should be formed in the organic phase where the nitrate binding to the uranyl changes from a monodentate to a bidentate form. Water is also co-extracted into the organic phase along with metal cations as we have previously reported in the uranyl case, and as experiments have demonstrated.4,6,7 Experimental studies have shown that water plays an important role in the microscopic structure formation and phase separation in the organic phase during the extraction process, ${ }^{6}$ thus it is important to understand at the fundamental level how water molecules leave the aqueous phase at the interface, what molecular 
processes take place, and in what form the extracted water exists in the bulk organic phase.

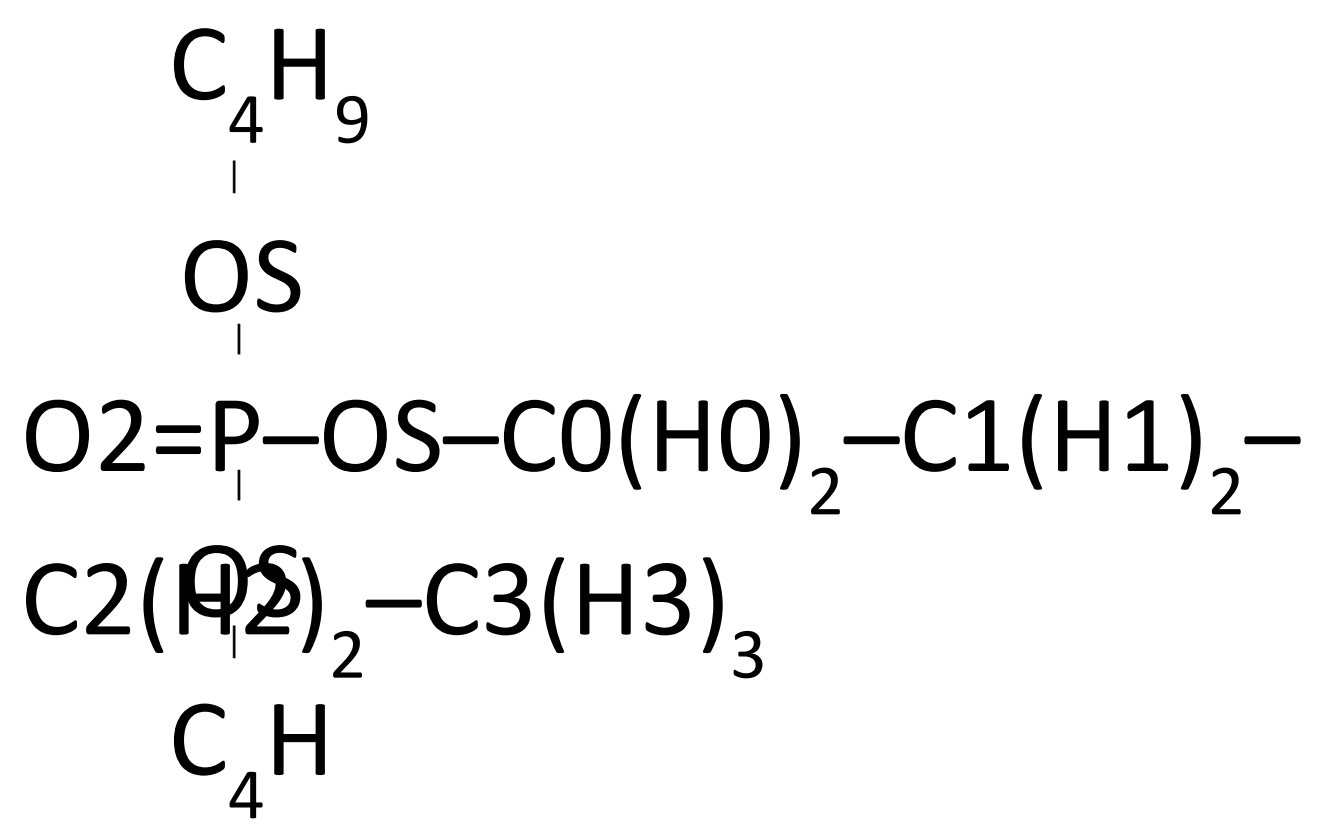

Schematic 1. The chemical structure of TBP molecule with the naming convention of atoms which are referred to in the text.

In published literature, several theoretical models have been proposed on the partitioning equilibria for acid and water extraction. ${ }^{8-14}$ However, all these thermodynamic models have to rely on assumed acid and water cluster composition in advance, and do not provide information on how water molecules transfer to the organic phase. Here, molecular dynamics simulations can provide molecular level insight and understanding complementary to experimental methods. Major challenges to obtaining accurate molecular level information of water extraction include development of realistic molecular models to predict a wide range of thermo-physical properties, and long-time simulations necessary to determine the extraction rate and the equilibrium solubility. In 
comparison with water and $n$-dodecane, TBP is less well studied and its force fields have thus not been extensively tested. In a prior study, we have examined several TBP force field models. ${ }^{15}$ However, the performance of these TBP force fields in modeling water extraction remains to be tested. Therefore, in this study, we investigate the performance of selected TBP force fields on water extraction to derive fundamental molecular level complexation mechanisms. The LAMMPS Molecular Dynamics Simulator ${ }^{16}$ was used to perform all simulations in this work.

\section{Methodology}

\subsection{Molecular Models}

Our simulation system consists of a pure water phase in direct contact with an organic mixture of TBP and $n$-dodecane. The concentration of TBP is approximately 30 vol\% which is a standard value in industrial applications. For water, we used a flexible SPC model. ${ }^{17}$ For TBP, we used a recently developed OPLS-DFT model ${ }^{15}$ and compared to the Amber-MNDO model. ${ }^{15}$ For $n$-dodecane, we used a re-optimized OPLS All-Atom force field with scaling factor at 0.4 .18 The parameters for cross interactions between different molecular species (atomic groups) were derived using the standard Lorentz-Berthelot rules. ${ }^{19}$ The particle-particle particle-mesh method, ${ }^{20}$ as implemented in LAMMPS, ${ }^{16}$ with a real space truncation distance of 15 $\AA$ and an accuracy of $10^{-6}$ was used to treat the electrostatic interactions.

\subsection{Molecular Dynamics Simulation Details}


We carried out molecular dynamics simulations of two-phase systems in the NPT ensemble at $25{ }^{\circ} \mathrm{C}$ and 1 bar running LAMMPS ${ }^{16}$ with the Verlet integrator and a Nosé-Hoover thermostat and barostat (with a temperature damping parameter of $0.1 \mathrm{ps}$, and a pressure damping parameter of $1 \mathrm{ps}$ ) using a time step of $1 \mathrm{fs}$. The initial aqueous and organic subsystems were created separately and equilibrated. The mass density of the organic subsystem was chosen to correspond to the experimental value of the TBP $/ n$-dodecane mixture at 30 vol\% TBP concentration. ${ }^{21}$ The initial configurations of both subsystems were created by placing molecules on lattice points in the simulation box with Lennard-Jones size parameters of the atoms set to zero and gradually increased to full size in a short MD simulation. An additional 4 ps run was performed for the pure water subsystem for equilibration. The bulk organic TBP/n-dodecane subsystem was equilibrated for 1.04 ns with TBP uniformly distributed in the dodecane diluent initially. Subsequently, the organic phase was slightly compressed in the direction orthogonal to the interface $(z$ direction) using two flat walls to avoid an overlapping problem when the two subsystems were combined. Finally, the two subsystems were combined into a single system by placing the aqueous system and the organic subsystem adjacent to each other in the $z$-direction. Initially, the organic subphase size was $86.019 \times$ $86.019 \times 81.724 \AA^{3}$ containing $1050 n$-dodecane and 450 TBP molecules. The pure water phase size for the system was $86.019 \times 86.019 \times 40.549 \AA^{3}$ containing 10,000 water molecules. The resulting total simulation box size was then $86.019 \times 86.019 \times$ $125.273 \AA^{3}$, taking into account the width of the interfaces. Periodic boundary conditions were used in all three dimensions. As a result, the system contained two 
interfaces. At the beginning of the simulation, the interfaces between the two subsystems were sharp. The combined system was subsequently evolved using a constant NVT ensemble for about 116 ps to obtain equilibrated aqueous-organic interfaces and bulk subphases. The system thus created was then used as the initial condition for NPT ensemble simulations using LAMMPS.

\section{Results and discussions}

\subsection{Water solubility as a function of TBP force field}

Figure 1 shows the water concentration as a function of time with two different TBP force fields. The system studied with the AMBER-MNDO TBP force field is 4 times larger in the interfacial area than that with the OPLS-DFT, with a total 358,800 atoms. This leads to less fluctuation in the results obtained with the former force field (Figure 1a) when compared to the latter (Figure 1b); especially in the plateau region. Clearly, water molecules in the organic phase for both systems have reached the saturation limit at approximately $40 \mathrm{~ns}$ and $80 \mathrm{~ns}$, respectively. The experimental solubility of water in the organic phase for the system is about 5.8 g/L. ${ }^{7}$ However, the saturated solubility calculated for the system with AMBERMNDO TBP force field is about $11 \mathrm{~g} / \mathrm{L}$, and for OPLS-DFT is about $12 \mathrm{~g} / \mathrm{L}$. We attribute this significant over-prediction to the relatively large dipole moment of the TBP force field. In our previous study ${ }^{15}$ we have established that these TBP models exhibit larger dipole moment than the experimentally measured value, 3.07-3.32 D. Specifically, the AMBER-MNDO force field gives rise to a dipole moment of 4.97 D while the OPLS-DFT force field predicts a dipole moment of $3.80 \mathrm{D}$, which is much 
closer to experiment than the former. Therefore, we have conducted a series of simulations using the OPLS-DFT-based model for TBP with slightly reduced atomic partial charges to investigate the influence of TBP dipole moment on the water extraction process.
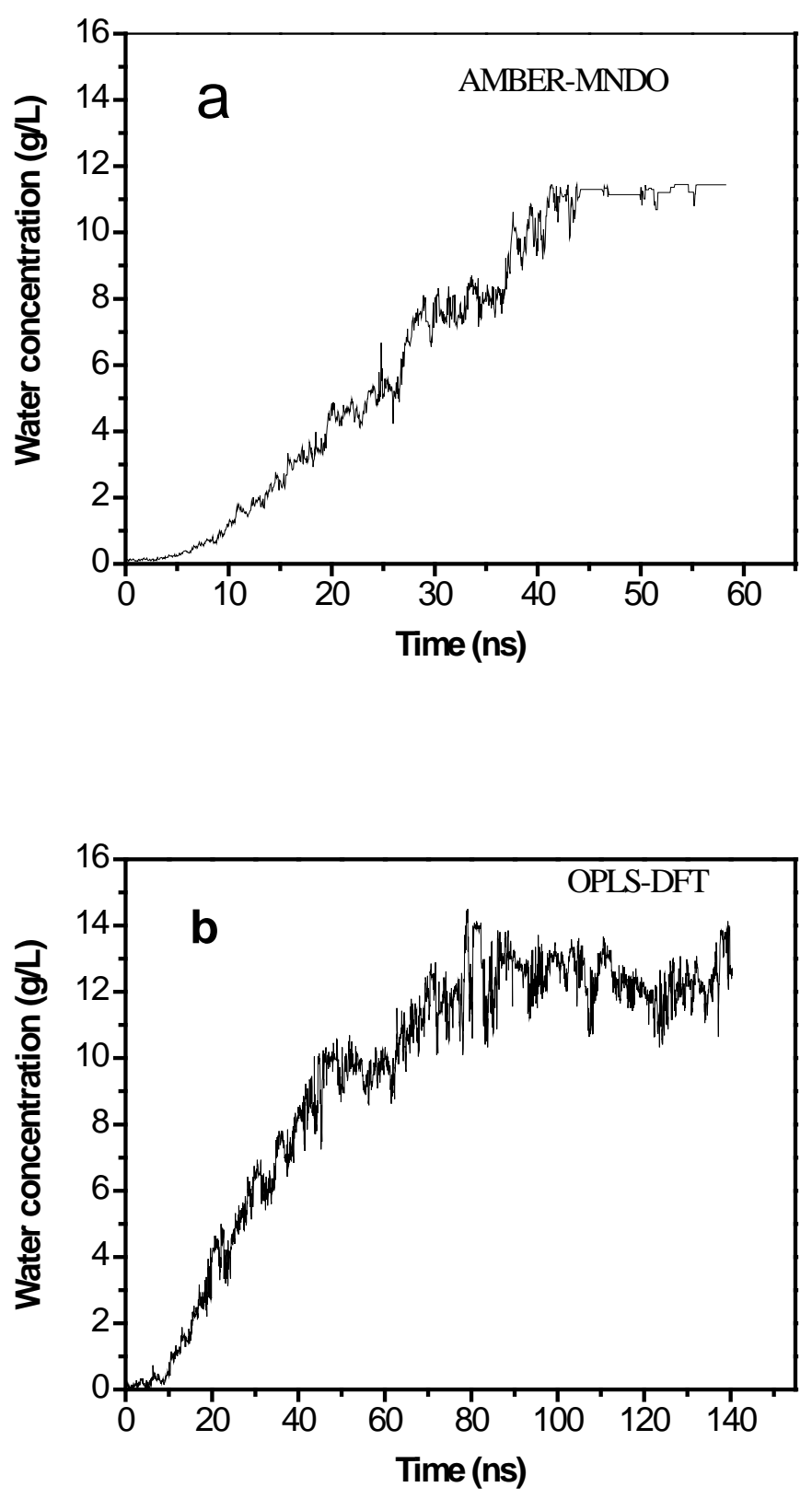
Figure 1. Comparison of water extraction for two different TBP force fields, namely, AMBER-MNDO and OPLS-DFT. (a) Water concentration in the organic phase as a function of time with AMBER force field and MNDO atomic partial charge distribution for TBP. (b) Water concentration in the organic phase as a function of time with OPLS force field DFT atomic partial charge distribution for TBP.

Figure 2 depicts water solubility in the organic phase as a function of time with the OPLS force field and DFT-based charge of TBP scaled by factors 0.9 and 0.8 , respectively. With TBP atomic partial charge scaled by 0.9 , the saturation water solubility is $4.5 \mathrm{~g} / \mathrm{L}$. However, with TBP atomic partial charge scaled by 0.8 , the saturated water solubility is less than $1 \mathrm{~g} / \mathrm{L}$. As the TBP atomic partial charge decreases, the dipole moment of TBP decreases monotonically. ${ }^{15}$ Comparing Figure $1 \mathrm{~b}$ and Figure 2, it is evident that a larger TBP dipole moment facilitates water transfer into the organic phase. Simulation with DFT-based atomic partial charge of TBP scaled by a factor 0.9 is in reasonable agreement with the experimentally measured solubility. Therefore, in the following analysis, we will mainly focus on the OPLS-DFT TBP force field with DFT-based atomic partial charges scaled by 0.9 unless otherwise noted. 


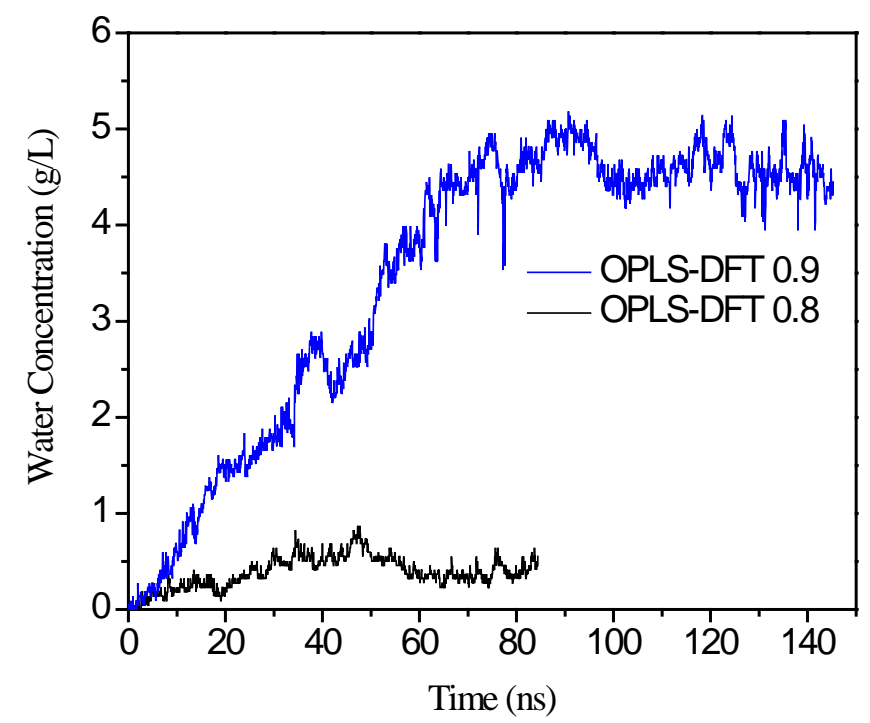

Figure 2. Water concentration in organic phase as a function of time with OPLS-DFT force field for TBP with the DFT-based atomic partial charge reduced by a factor 0.9 , and 0.8 , respectively.

\subsection{Water extraction mechanisms}

\section{(1) Interfacial roughness}

Figure 3 shows water (based on oxygen atom position) and TBP (based on phosphorus atom position) profiles across the simulation box for two different TBP atomic partial charge distributions: one with DFT-based charge scaled by 0.8 (corresponding to a dipole moment $3.31 \mathrm{D}^{15}$ ), and the other with the original DFTbased charge. Comparing these two TBP models, the system with higher TBP dipole moment has a wider peak at the interface, and thus a wider interface and a larger amount of water in contact with the organic phase. Examining the corresponding TBP profiles in the bulk region, it is seen that the system with higher TBP dipole moment has a lower profile. Therefore, the system with higher dipole moment has 
fewer TBP molecules in the bulk region and hence more TBP molecules in the interfacial region.

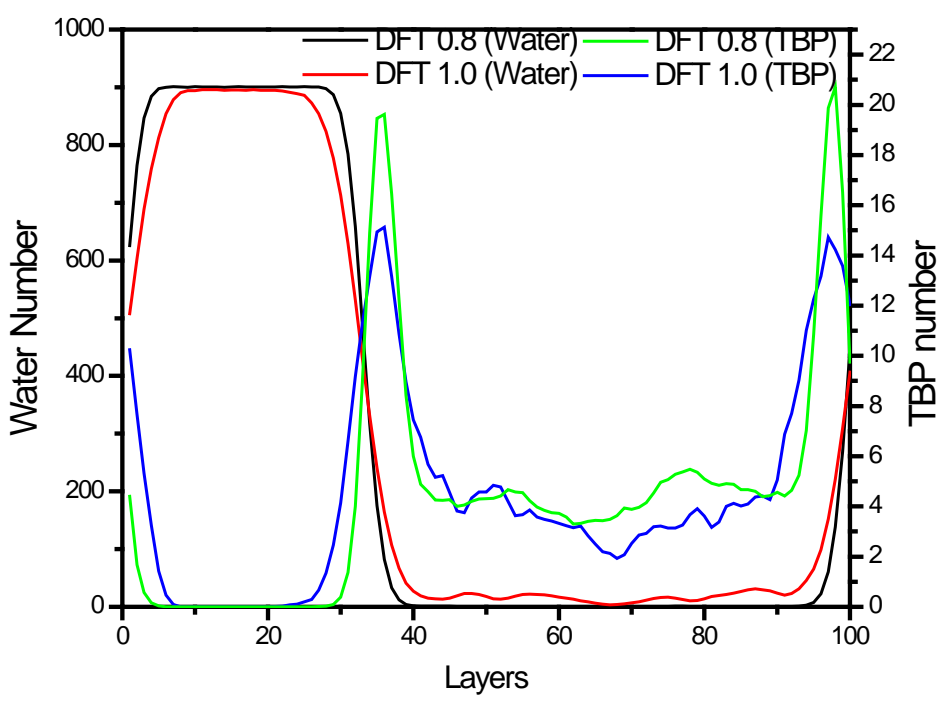

Figure 3. Water (based on the oxygen atom position) and TBP (based on the phosphorus atom position) profiles across the simulation box with two different TBP atomic charge values. Note the widening of the interface and the lower bulk TBP profile for OPLS-DFT model with larger dipole moment.

Further examination of the configurations of the interface (Figure 4) shows that the system with higher TBP dipole moment induces larger interfacial roughness. When the interface roughness is larger, the effective interfacial area is larger, and more water molecules are exposed to the organic phase. Furthermore, perhaps more importantly, more water molecules are located at the edges or tips of the aqueous-organic interface with fewer hydrogen bonds connected to the aqueous phase. Thus, with larger interfacial roughness, water molecules have a higher probability of breaking away from the aqueous phase and hence a higher probability 
of entering the organic phase. Since the interface roughness is determined by the dipole moment of TBP, the atomic partial charge distribution in the TBP molecule has a significant effect on the rate of water extraction into the organic phase by virtue of hydrophilic interactions with the aqueous phase. These interactions modulate the ability of TBP molecules to replace water-water hydrogen bonding at the interface with water-TBP binding (through the 02 atom) to weaken the connection of the interfacial water with the aqueous bulk. Thermal fluctuations help complete the process of breaking the remaining hydrogen bonds with bulk water allowing for water extraction in the organic phase. Our results indicate that the critical step in the extraction process is the breaking of hydrogen bonds of interfacial water molecules with the aqueous phase. In the following we will discuss this hydrogen bond breaking process in details.
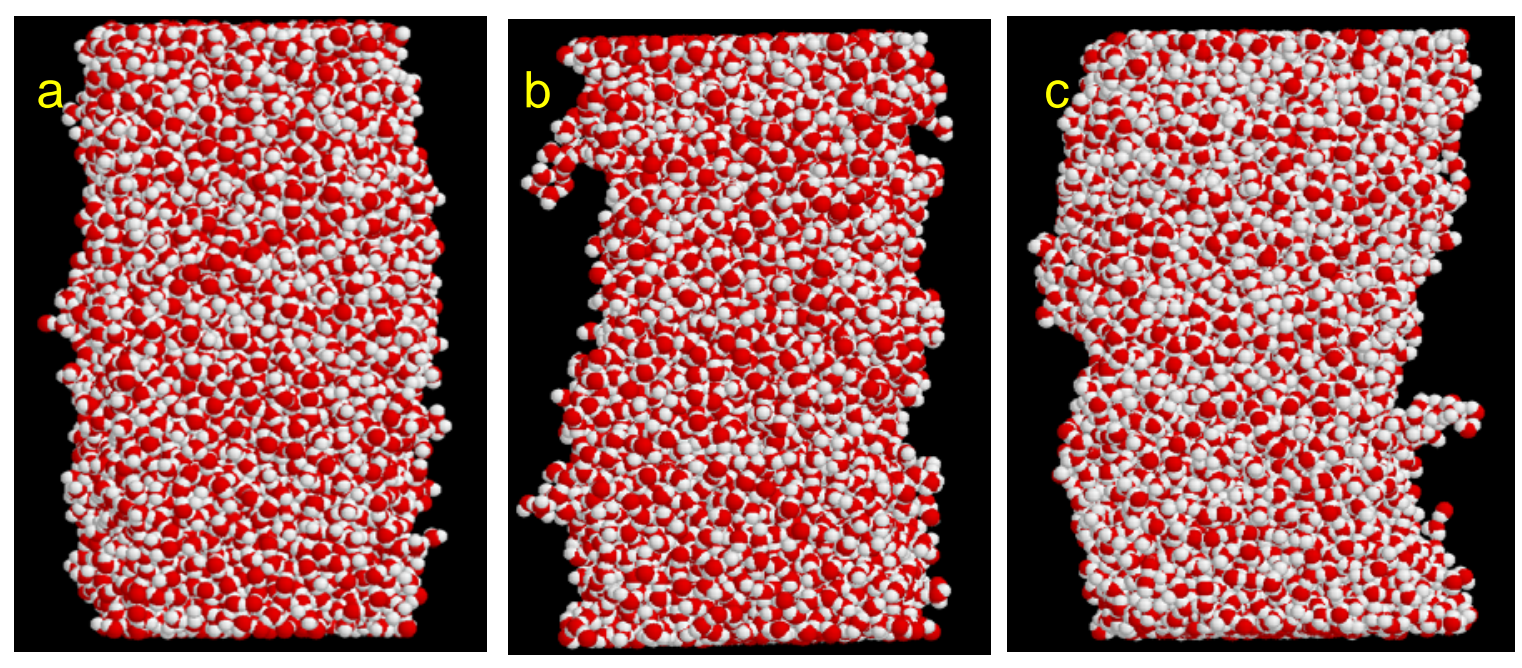

Figure 4. Snapshots showing the interfacial roughness for the OPLS-DFT model. (a) atomic charges scaled by 0.8 at $84.485 \mathrm{~ns}$. (b) scaled by 0.9 at $145.520 \mathrm{~ns}$. (c) original charges at 1490.095 ns The oxygen of water is in red, and the hydrogen of 
water is in white. TBP, $n$-dodecane, and water without hydrogen bonds are not shown in the snapshots.

\section{(2) Molecular pathways of water extraction}

To better understand the simulation results of the water extraction process, we closely tracked the entire extraction processes of some of the water molecules. In a series of snapshots (Figure 5 to Figure 8), we show the bulk water, the tracked water molecules (for which the oxygen atom is colored in green), the water molecules already in the organic phase that form a cluster with the tracked water molecules, and TBP molecules which form hydrogen bonding with a tracked water molecule or a cluster of tracked water molecules. Figure 5 shows water extracted as a single molecule. The water molecule was initially (80.030 ns) at the interface, with the oxygen atom of the water bonded to bulk water through two hydrogen bonds. At the same time, one of the two hydrogen atoms formed a hydrogen bond with one TBP (Figure 5a). Subsequently, one of the two hydrogen bonds of the oxygen with bulk water is broken while the hydrogen atom of the water molecule forms a weak hydrogen bond with an OS atom of a nearby TBP molecule (Figure 5b). The remaining single hydrogen bond with bulk water is not strong enough to sustain the connection, consequently the water molecule breaks off the aqueous bulk phase and moves into the organic phase (Figure 5c-d). It is important to note that the extracted water molecule is hydrogen bonded to a TBP molecule during the entire process, providing some of the driving force for pulling the water molecules into the organic phase. 

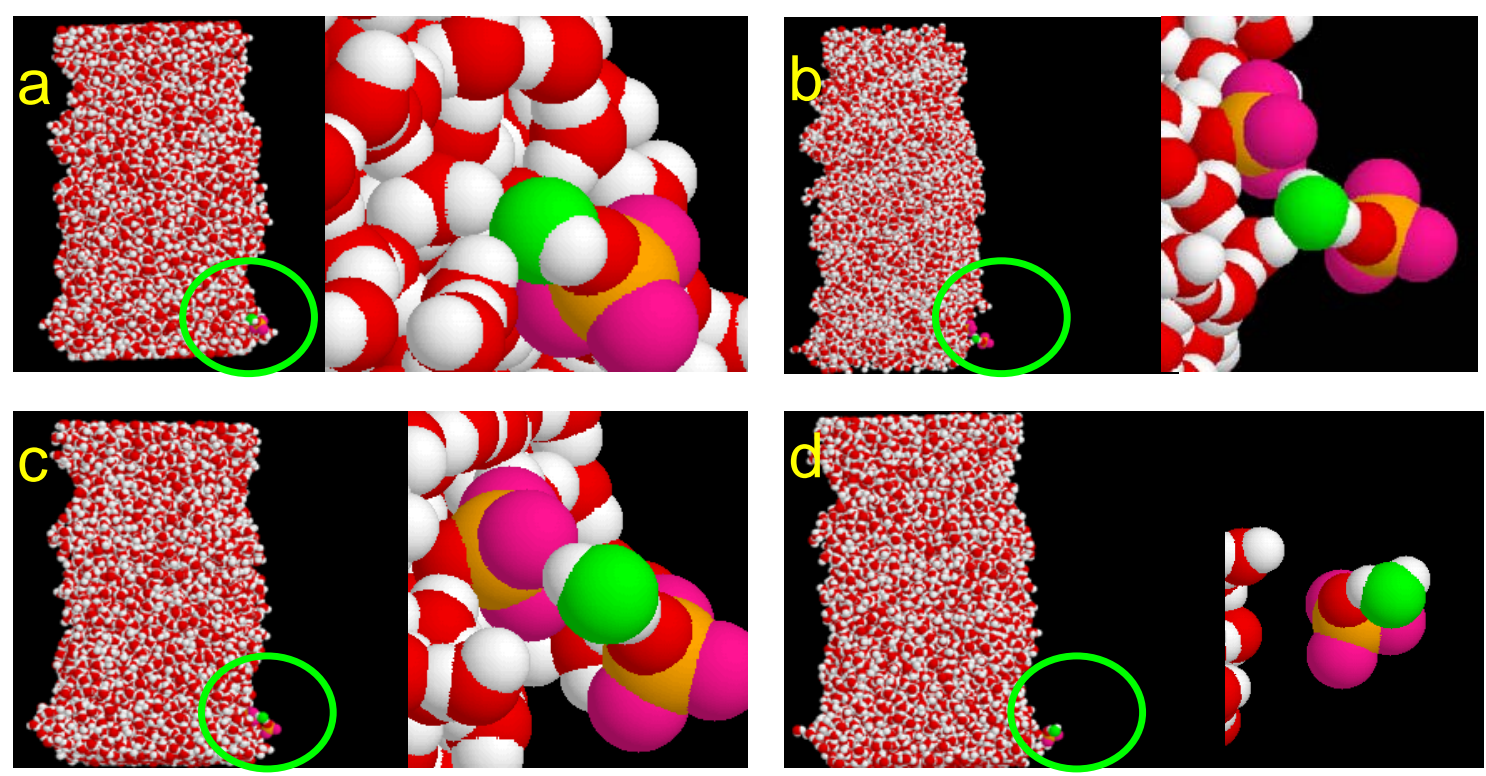

Figure 5. Water extracted as a single water molecule by one TBP molecule with the assistance of another TBP molecule. Snapshots at: (a) $80.030 \mathrm{~ns}$, (b) $80.040 \mathrm{~ns}$, (c) $85.045 \mathrm{~ns}$, and (d) 80.050ns. The oxygen of the water molecule being tracked is in green, and the hydrogen of the tracked water is in white. Otherwise, the oxygen of water is in red, and the hydrogen of water is in white. For TBP, only the TBP head group is shown, 02 is in red, $\mathrm{P}$ is in yellow, and OS is in pink. In all snapshots, $n$ dodecane is not shown.
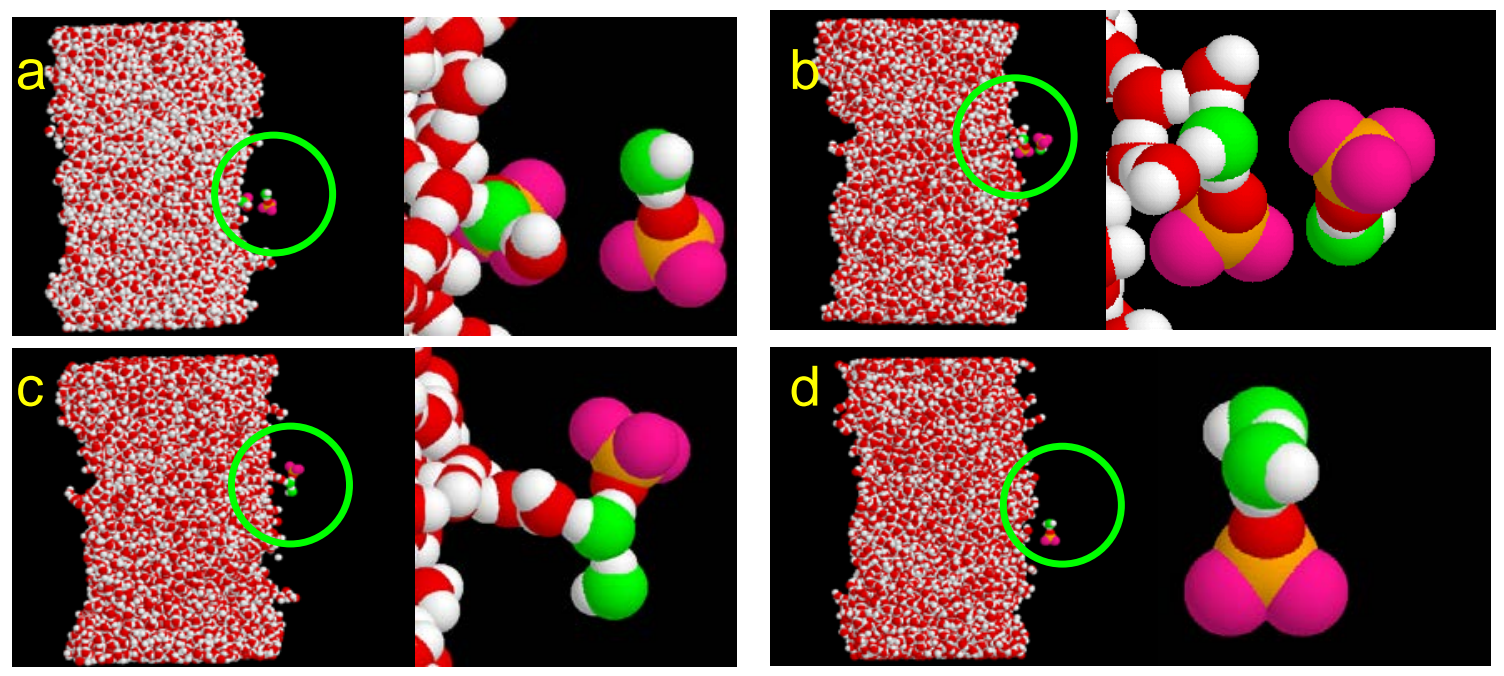
Figure 6. Water extracted as a two-molecule cluster carried by one TBP molecule. Snapshots at: (a) $31.500 \mathrm{~ns}$, (b) $31.550 \mathrm{~ns}$, (c) $31.600 \mathrm{~ns}$, and (d) $31.700 \mathrm{~ns}$. Same color codes as in Figure 5.
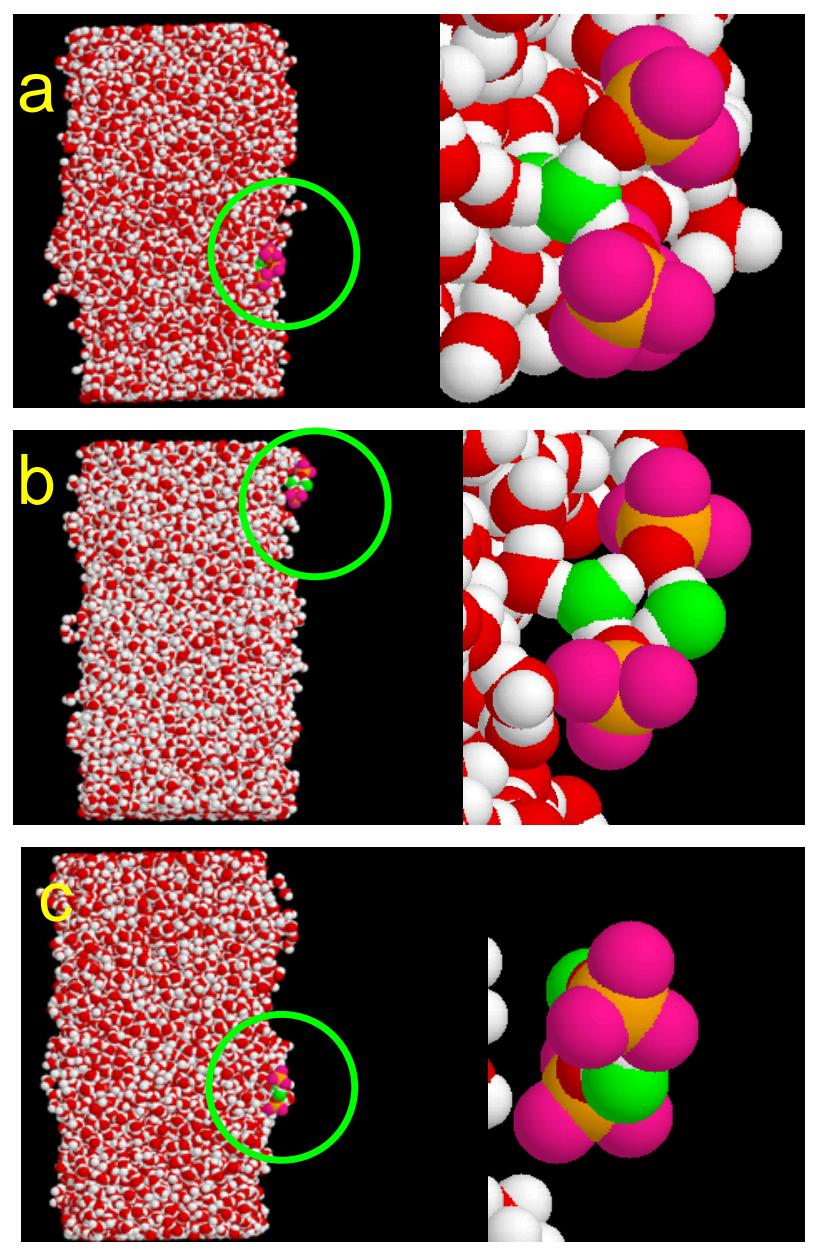

Figure 7. Water extracted as two water molecules and two TBP molecules. Snapshots at: (a) $10.750 \mathrm{~ns}$, (b) $10.800 \mathrm{~ns}$, and (c) $10.850 \mathrm{~ns}$. Same color codes as in Figure 5. 

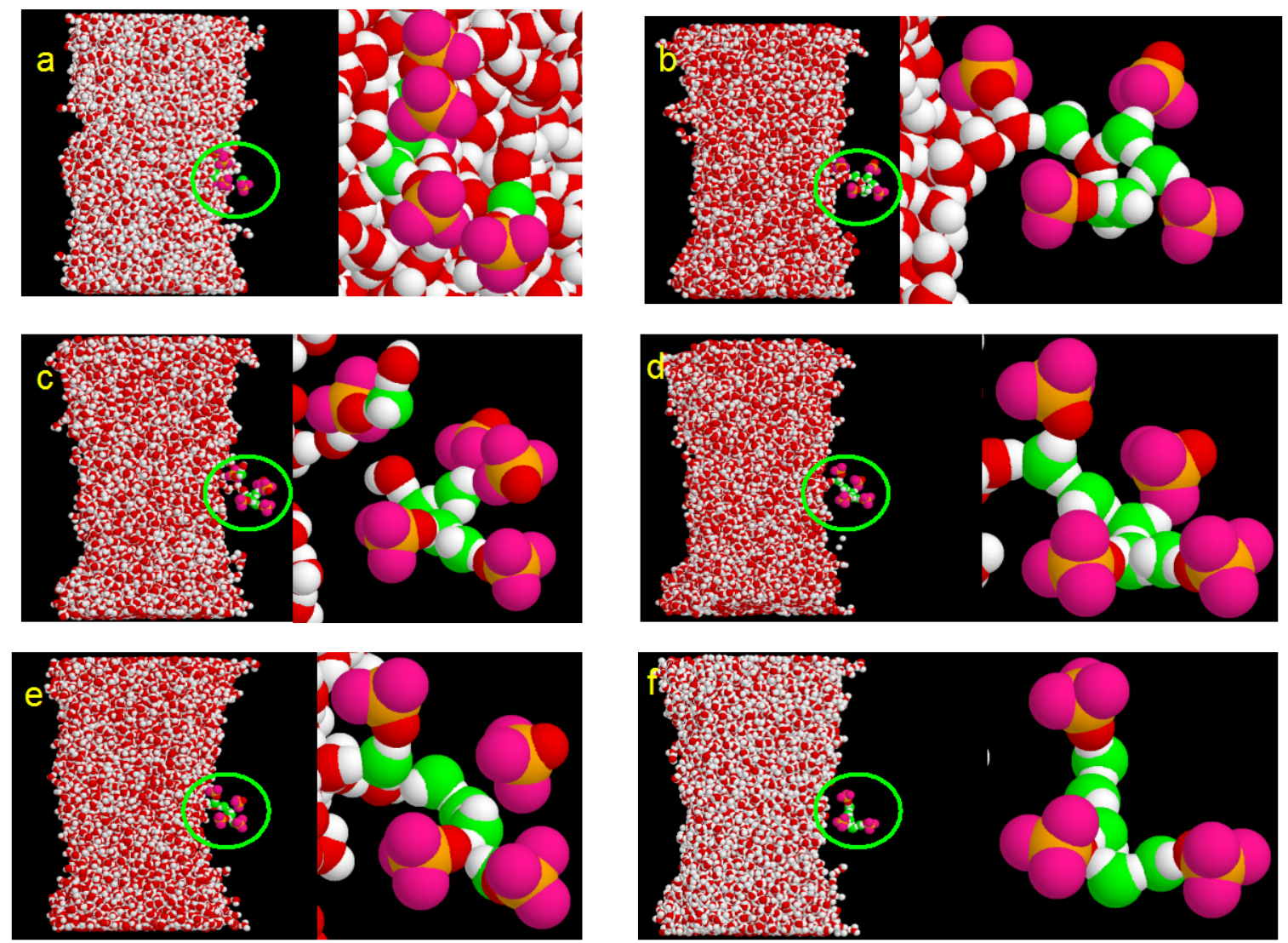

Figure 8. Water extracted as a cluster. Snapshots at: (a) $84.900 \mathrm{~ns}$, (b) $85.100 \mathrm{ns,} \mathrm{(c)}$

$85.110 \mathrm{~ns}$, (d) $85.150 \mathrm{~ns}$, (e) $85.155 \mathrm{~ns}$, and (f) $85.160 \mathrm{~ns}$. Same color codes as in Figure 5.

Water can also be extracted as a two-molecule cluster with one TBP bonded to it as shown in snapshots in Figure 6. In Figure 6a, one of the tracked water molecules is already in the organic phase, and the other tracked water molecule is still hydrogen bonded with bulk water (through one hydrogen of the water) while the other hydrogen of the same water forms a hydrogen bond with a TBP. In Figure $6 \mathrm{~b}$, the interfacial water moves further into organic phase, however, it is still connected with bulk water through two hydrogen bonds while bound with TBP. As the two tracked water molecules move closer (shown in Figure 6c), they are 
hydrogen bonded together, and a water molecule chain is formed. This hydrogen bond chain, however, is not stable. Therefore, the two tracked water molecules break their hydrogen bonding with bulk water as a two-water molecule cluster bound with one TBP.

Water has also been extracted as a cluster of two water molecules and two TBP as shown in Figure 7. In Figure 7a, two TBP form hydrogen bonds with one tracked water molecule, which has two hydrogen bonds with bulk water with another tracked water molecule close by. The two TBP eventually pull the two tracked water molecules further into the organic phase, there is then only one remaining hydrogen bond with bulk water, as shown in Figure 7b. Similarly to the single water molecule extraction, this single hydrogen bond is easy to break up, and the two tracked water molecules are extracted together into the organic phase by two TBP as a cluster.

Water can also be extracted as a larger cluster as shown in Figure 8, where five water molecules are being tracked. Initially (at $84.900 \mathrm{~ns}$, Figure 8a), one tracked water molecules is already in the organic phase, and the other four interfacial water molecules are hydrogen bonded to bulk water. Subsequently (at $85.100 \mathrm{~ns}$, Figure 8b), the four water molecules further move into the organic phase (still have remaining hydrogen bonds with bulk water), and form a molecular chain with the earlier tracked water molecule that was already in the organic phase at 84.900 ns. However, such a long molecule chain is very weakly connected within itself and with the bulk water. Thus, at 85.110 ns (Figure 8c) the long molecular chain breaks up into two clusters while moving into the organic phase. One cluster 
contains two water molecules and one TBP, the other cluster contains five water molecules and four TBP molecules. Next, at 85.150 ns (Figure 8d) and 85.155 ns (Figure 8e), the two water clusters form a new shorter water chain that reconnects with bulk water because there are not enough TBP in the neighborhood to stabilize the water clusters in the organic phase. Afterward, the water is extracted into the organic phase as a five-water-molecule cluster at $85.160 \mathrm{~ns}$ (Figure 8e).

To summarize, the simulated water extraction process is as follows. First, the interface has local microscopic roughness because of thermodynamic fluctuations. Second, TBP reduces the interfacial tension, and further enhances the interfacial roughness, which facilitates more water exposure to the organic phase. Therefore, effectively more TBP molecules are available at the interface forming hydrogen bonds with interfacial water molecules promoting water molecules transfer into the organic phase. Thus, TBP has the dual role of reducing interfacial tension as well as bonding to water hydrogen to prevent the water molecule reconnecting to the bulk aqueous phase. This provides the driving force for water transfer to the organic phase. Last but not least, local microscopic roughness creates various sized local protrusion of water molecules into the organic phase, allowing the exposed water molecules to be extracted as various sized clusters or as a single water molecule.

A final note is in order. The particular pathways and structural forms of water extraction are not limited to those discussed in the foregoing and the details may vary substantially. Nevertheless, it is demonstrated that realistic solubility values can be obtained at equilibrium, and an equilibrium mass-action based 
mechanism can be derived from these molecular level simulations; the latter is also of practical importance for unit operations modeling.

\subsection{Water cluster evolution in the organic phase}

Figure 9 shows water cluster size distributions in the organic phase as a function of time during the water extraction process. At the early stage of water extraction, about $50 \%$ of extracted water exists in organic phase as a single water molecule. Gradually, the percentage of the single water molecules in the organic phase decreases to between $20 \%$ and $30 \%$ when the water extraction process reaches equilibrium. These results clearly show that water molecules are more likely to form clusters in the organic phase.

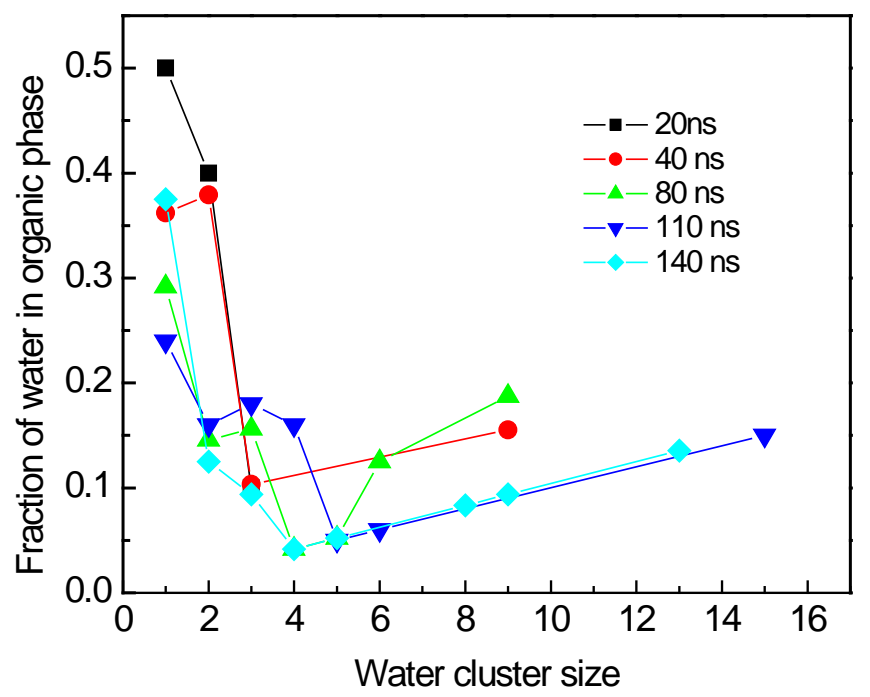

Figure 9. Water distributions in the organic phase as a function of time during the extraction process. The data indicate the tendency of water formation process in the 
organic phase. This process eventually reaches equilibrium as indicated by the distribution at 110 and $140 \mathrm{~ns}$.

However, the water clusters in the organic phase are usually small as shown in Figure 10. We analyzed the water cluster distribution from about $80 \mathrm{~ns}$, when it first reaches equilibrium, to the end of the simulation at about $140 \mathrm{~ns}$. The average total number of water molecules in the organic phase is about 100 . There exist 27 water clusters as a single molecule, 19 water clusters as two molecules, and 12 water clusters as three molecules in the organic phase. Integration of the water number in Figure 10 clearly shows that the majority of water molecules form clusters, and that the majority of these water clusters have less than 5 water molecules.

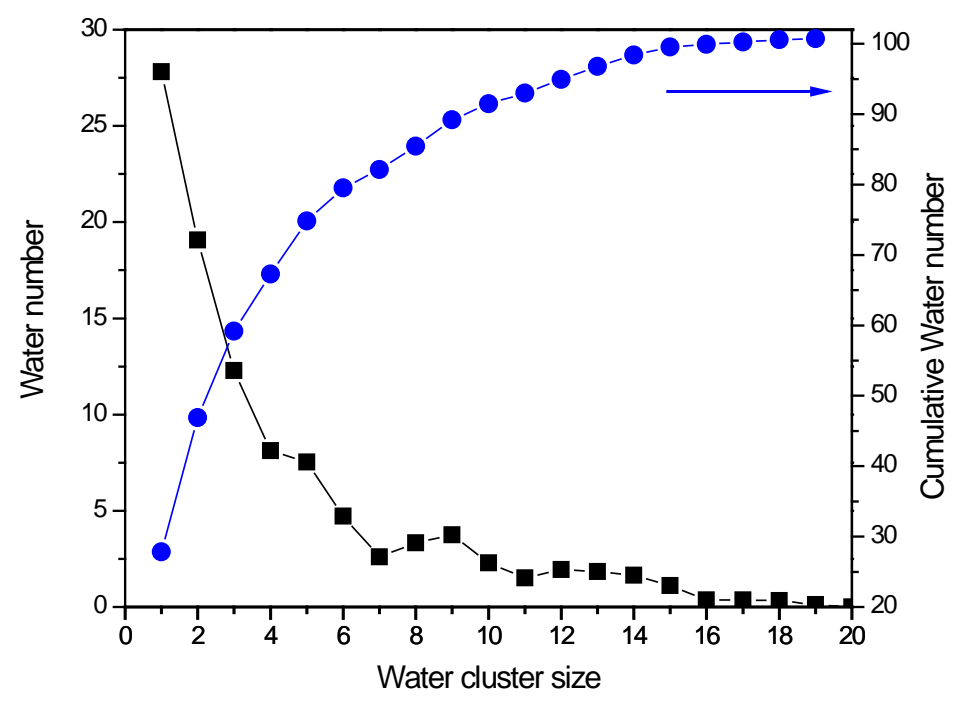

Figure 10. Average water distributions (left axis) in the organic phase after the water extraction process has reached equilibrium (between $80-140 \mathrm{~ns}$ ) and the cumulative number of water molecules as a function of cluster size (right axis). 


\section{Conclusions}

Molecular dynamic simulations have been performed to investigate water extraction by $30 \mathrm{vol} \%$ TBP in $n$-dodecane. It is found the TBP electric dipole moment has a significant effect on the predicted water solubility. By scaling down the original DFT-based atomic partial charges of the TBP molecular model by $0.9,15$ water solubility close to that measured in experiments was predicted.

It is found that a higher TBP dipole moment increases the roughness of the aqueous-organic interface as a result of reduced interfacial tension (or equivalently, the decreased free energy required for interface area expansion). Because of the rougher interface, more water molecules are exposed to the organic phase, and are located at the edges or tips of the aqueous-organic interface with fewer hydrogen bonds connected to the aqueous phase. This creates a favorable environment for water hydrogen bond breaking with the bulk water and subsequent transfer to the organic phase.

Our simulation results indicate that in the water extraction process, the critical step is the hydrogen bond breaking of interfacial water molecules. In this step, TBP plays a critical role in a) forming hydrogen bond with hydrogen atoms of interfacial water, b) preventing water molecules' dangling bonds to reconnect with bulk water, and c) providing a driving force for finally breaking the weakly bonded single hydrogen bond of water or water clusters. Thus, the interface coverage by TBP molecules is crucial both in increasing the interfacial roughness and providing the driving force for extracting water into the organic phase. This work suggests 
that in the design of extracting agents, it is important to consider their influence on interfacial properties, when considering practical applications.

This is the first application of molecular modeling and simulation to provide insight and quantitative information on the transport of water into a solvent extraction solution of industrial interest. The solubility of water in a common mixture of TBP/dodecane obtained by our simulation is satisfactorily close to experimental data. At the molecular level, unique insight about the transfer of water molecules into the organic phase was obtained. For instance, water molecules are extracted as single water molecules or as various sized water clusters with TBP attached to them. After entering into the organic phase, most of the extracted water exists in the form of small water clusters with the majority of them containing less than 5 water molecules at saturation equilibrium. In summary, this work paves the way for the use of molecular simulation tools in hydrometallurgy and nuclear solvent extraction areas wherein future extensions to investigate more complex aqueous phases, including metal ions, is now at reach.

\section{Acknowledgement}

This work was supported by the US Department of Energy, Office of Nuclear Energy under the Nuclear Energy University Program (DOE-NEUP), contract number: DEAC07-051D14517. Computing resources used at the Center for Advanced Modeling and Simulation at the Idaho National Laboratory in collaboration with the Nuclear Energy Advanced Modeling and Simulation (NEAMS) program, and the Fuel Cycle Technology Separations and Waste Forms Campaign of the Nuclear Energy Office of DOE are greatly 
appreciated. The Oak Ridge National Laboratory is managed by UT-Battelle, LLC for the DOE under contract No. DE-AC05-00OR22725. 


\section{References}

(1) Rydberg, J.; Cox, M.; Musikas, C.; Choppin, G. R. Solvent Extraction Principles and Practice, , 2nd ed. Revised and Expanded ed.; New York : M. Dekker 2004.

(2) Nash, K. L.; Madic, C.; Mathur, J. N.; Lacquement, J. Actinide Separation Science And Techology. In The Chemistry of the Actinide and Transactinide Elements Dordrecht : Springer 2006; pp 2622.

(3) Ye, X.; Smith, R. B.; Cui, S.; de Almeida, V.; Khomami, B. Solvent Extraction and Ion Exchange 2010, 28, 1.

(4) Ye, X.; Cui, S.; de Almeida, V. F.; Hay, B. P.; Khomami, B. Physical Chemistry Chemical Physics 2010, 12, 15406.

(5) Ye, X.; Cui, S.; de Almeida, V. F.; Khomami, B. Journal of Physical Chemistry B 2009, 113, 9852.

(6) Chiarizia, R.; Jensen, M. P.; Borkowski, M.; Ferraro, J. R.; Thiyagarajan, P.; Littrell, K. C. Solvent Extraction and Ion Exchange 2003, 21, 1.

(7) Schulz, W. W.; Navratil, J. D. Science and technology of tributyl phosphate; CRC Press: Boca Raton, FL, 1984; Vol. 1.

(8) Alcock, K.; Grimley, S. S.; Healy, T. V.; Kennedy, J.; McKay, H. A. C. Transactions of the Faraday Society 1956, 52, 39.

(9) Hlushak, S. P.; Simonin, J. P.; Caniffi, B.; Moisy, P.; Sorel, C.; Bernard, O. Hydrometallurgy 2011, 109, 97.

(10) Blaylock, C. R.; Tedder, D. W. Solvent Extraction and Ion Exchange $1989,7,249$. 
(11) Chaiko, D. J.; Vandegrift, G. F. Nuclear Technology 1988, 82, 52.

(12) Davis, W. New England Journal of Medicine 1962, 267, 174.

(13) Naganawa, H.; Tachimori, S. Bulletin of the Chemical Society of Japan 1997, 70, 809.

(14) Ziat, K.; Mesnaoui, B.; Bounahmidi, T.; Boussen, R.; de la Guardia, M.; Garrigues, S. Fluid Phase Equilibria 2002, 201, 259.

(15) Cui, S.; de Almeida, V. F.; Hay, B. P.; Ye, X.; Khomami, B. Journal of Physical Chemistry B 2012, 116, 305.

(16) Plimpton, S. Journal of Computational Physics 1995, 117, 1.

(17) Wu, Y. J.; Tepper, H. L.; Voth, G. A. Journal of Chemical Physics 2006, $124,024503$.

(18) Ye, X.; Cui, S.; de Almeida, V. F.; Khomami, B. Journal of Molecular Modeling 2013, 19, 1251.

(19) Martin, M. G.; Siepmann, J. I. Journal of Physical Chemistry B 1998, 102, 2569.

(20) Darden, T.; York, D.; Pedersen, L. Journal of Chemical Physics 1993, 98, 10089.

(21) Tian, Q. L.; Liu, H. Z. Journal of Chemical and Engineering Data 2007, 52, 892. 